\title{
Locomotor improvement of spinal cord-injured rats through treadmill training by forced plantar placement of hind paws
}

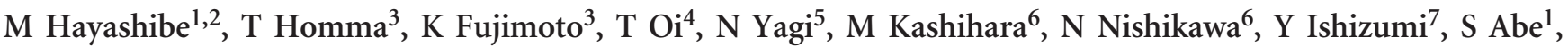

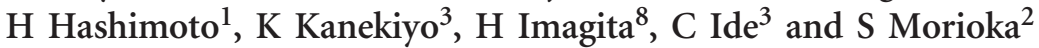

Study design: Experimental training model of rats with spinal cord injury (SCI).

Setting: Osaka, Japan

Objective: To investigate the effect of forced treadmill training by plantar placement (PP), as compared with dorsal placement (DP), of the dorsal paws on the locomotor behaviors of spinal cord-injured rats.

Methods: The spinal cord was contusion-injured at the thoracic level. Rats were divided into three groups: forced training involving stepping by PP and DP and non-forced training/assistance (nT). Training began 1 week after injury and was conducted for 4 weeks. Locomotor behaviors were estimated using Basso-Beattie-Bresnahan (BBB) scores, dorsiflexion of the hind paws and footprints of the hind paws. Histological and immunohistochemical examinations of the spinal cord lesions were conducted after 4 weeks of training. Results: The values, respectively, of PP, DP and nT groups at 4 weeks of training were as follows: BBB scores were $15.6 \pm 0.8$, $7.7 \pm 1.3$ and $10.3 \pm 0.4$. The paw dorsiflexion angles were $34.1 \pm 5.2,16.4 \pm 2.4$ and $23.6 \pm 3.0$ degrees, respectively. The stride angles were $5.1 \pm 0.9,13.7 \pm 4.9$ and $17.8 \pm 4.0$ degrees for the left paws. Cavity volumes were $10.3 \pm 2.1,31.0 \pm 2.0$ and $28.2 \pm 4.9 \%$. In addition to cavities, there were astrocyte-devoid areas containing some loose tissues, through which many axons extended longitudinally.

Conclusions: The BBB score, dorsiflexion angle and stride angle were consistently improved in the PP group. Cavity formation was more reduced, and many axons extended through coarse tissues formed in astrocyte-devoid areas at the lesion in the PP group. Forced training by PP of the hind paws promoted the behavioral and histological improvement of rats with $\mathrm{SCl}$.

Spinal Cord (2016) 54, 521-529; doi:10.1038/sc.2015.186; published online 20 October 2015

\section{INTRODUCTION}

Spinal cord injury (SCI) is devastating; patients with SCI lose functions below the level of the injury, and recovery of the locomotor functions is limited even with extensive rehabilitation. Body weight support treadmill (BWST) training with plantar stepping is widely used for locomotor exercises in the rehabilitation of patients with SCI. However, the effectiveness of plantar stepping of the hind feet has not been fully evaluated experimentally. The present study focused on the effect of forced treadmill training by plantar placement (PP) of the hind paws on the locomotor behaviors of rats with SCI. Recent cell transplantation studies have reported the recovery of injured spinal cord tissue and the behavioral improvement of SCI animals. ${ }^{1}$ Our previous studies showed that bone marrow stromal cell transplantation is effective for locomotor recovery from SCI in rats, ${ }^{2}$ findings that have been applied to clinical cases. ${ }^{3}$ Along with the emergence of potentially effective treatments of SCI, including cell transplantation therapy, hope for the rehabilitation of patients with SCI has increased. It is now all the more important to experimentally explore the effects of rehabilitation on recovery from SCI.
Previously, rehabilitation mainly focused on training to utilize muscle functions above the level of the injury to improve activities of daily living. However, since the $1990 \mathrm{~s}$, BWST training has been developed for rehabilitation to promote locomotor abilities, including muscle functions below the level of the injury. ${ }^{4}$ The effects of locomotor training have been examined and assessed using laboratory animals. ${ }^{5}$ Battistuzo et al. ${ }^{6}$ reviewed studies of experimental locomotor training, referring to the marked heterogeneity in training paradigms and methods for assessing the level of recovery from SCI in laboratory experiments.

Dietz et al. ${ }^{7}$ reported that sensory inputs and body weight loading are crucial for the improvement of locomotor behaviors. Body weight loading on the legs is important for the locomotor training of patients. ${ }^{7,8}$ Plantar cutaneous sensation or sensory feedback from the hind paw has been shown to have an important role in the maintenance of normal rat locomotion. ${ }^{9}$ This means that sensory inputs from the hind limbs including the paw soles are important for repairing locomotor circuitry in the presence of a severe lesion of the spinal cord.

\footnotetext{
${ }^{1}$ Department of Occupational Therapy, Aino University School of Health Sciences, Osaka, Japan; ${ }^{2}$ Department of Neurorehabilitation, Graduate School of Health Sciences, Kio University, Nara, Japan; ${ }^{3}$ Institute of Regeneration and Rehabilitation, Aino University School of Health Sciences, Osaka, Japan; ${ }^{4}$ Department of General Education, Wakayama University, Wakayama, Japan; ${ }^{5}$ Department of Rehabilitation, Takatsuki Red Cross Hospital, Osaka, Japan; ${ }^{6}$ Department of Rehabilitation, Kyoto Kaisei Hospital, Kyoto, Japan; ${ }^{7}$ Department of Rehabilitation, North Osaka Police Hospital, Osaka, Japan and ${ }^{8}$ Department of Exercise and Functional Physiology, Graduate School of Health Sciences, Kio University, Nara, Japan

Correspondence: M Hayashibe, Department of Occupational Therapy, Aino University School of Health Sciences, Higashiohda 4-5-4, Ibaraki City, Osaka 567-0012, Japan. E-mail: m-hayashibe@ot-u.aino.ac.jp

Received 12 March 2015; revised 26 August 2015; accepted 4 September 2015; published online 20 October 2015
} 
In treadmill training of rats with SCI, hind limb movement is poor, with only slight or no flexion of hind limb joints in the early stages. Rats cannot perform PP of their hind paws until reaching a score of 9 on the 21-point Basso-Beattie-Bresnahan (BBB) score. It is reasonable to consider that the normal pattern of plantar stepping might be important for locomotor training in rats. This means that forced PP of hind paws may be beneficial during treadmill training in rats with SCI. Although a small number of studies referred to the $\mathrm{PP}$ of animals during locomotor training, ${ }^{10}$ many other studies gave no details concerning the pattern of paw placement in treadmill training.

The present study examined the effects of PP (that is, stepping with the paw sole placed by the examiner on the floor) of hind paws in BWST training in SCI rats. Comparison was made between stepping with PP and that with dorsal placement (DP; that is, stepping with the back (dorsum) of the paw and toes placed by the examiner on the floor) of hind paws. From the early stages of SCI, rats were forced by an examiner to place their paw sole in the PP group, or back of the paw and toes in the DP group, on the treadmill belt. Forepaws of rats were placed with their sole in contact with the treadmill belt.

The effects of treadmill training have usually been compared with those of non-training. Therefore, PP, DP and nT groups were prepared in the present study.

\section{MATERIALS AND METHODS}

\section{Experiment design}

Forty-five Sprague-Dawley rats (6-week-old, female) were purchased from an animal dealer (Klea, Osaka, Japan). Rats were acclimated for 1 week before surgery.

We preferred to use young adult rats, ${ }^{1,2}$ expecting that they could recover from the SCI faster than older adult rats.

Rats were maintained in conventional cages with a temperature of $23^{\circ} \mathrm{C}$, humidity of $55 \%$ and a 12 -h light and dark cycle. The study was carried out with approval of the Animal Committee of Aino University, and all animal experiments were performed in accordance with the Guidelines for Animal Experiments of Aino University based on the Guidelines for Proper Conduct of Animal Experiments and Related Activities formulated by the Japanese Ministry of Education, Culture, Sports, Science and Technology.

Two rats showing a score of 5 or more on the BBB score within 1 week after surgery were excluded from the experiment. Five rats had died before the start of training. Therefore, 33 rats were used for the training experiment. Rats were divided into three groups: treadmill training by PP $(n=11)$, treadmill training by DP $(n=11)$ and non-forced training/assistance (nT; $n=11)$. Five rats were assigned to the sham-operated group. Rats for treadmill training were acclimated to the moving treadmill by making them walk on it for $20 \mathrm{~min}$ every day for 1 week before surgery.

For the assessment of locomotor activities, the BBB score ${ }^{11}$ and stepping parameters, including the step length, step width and stride angle, ${ }^{5}$ were used. The dorsiflexion angle of the hind paws was estimated from a video recording. The histological and immunohistochemical findings of tissue recovery and axonal outgrowth at the lesion were also examined.

a

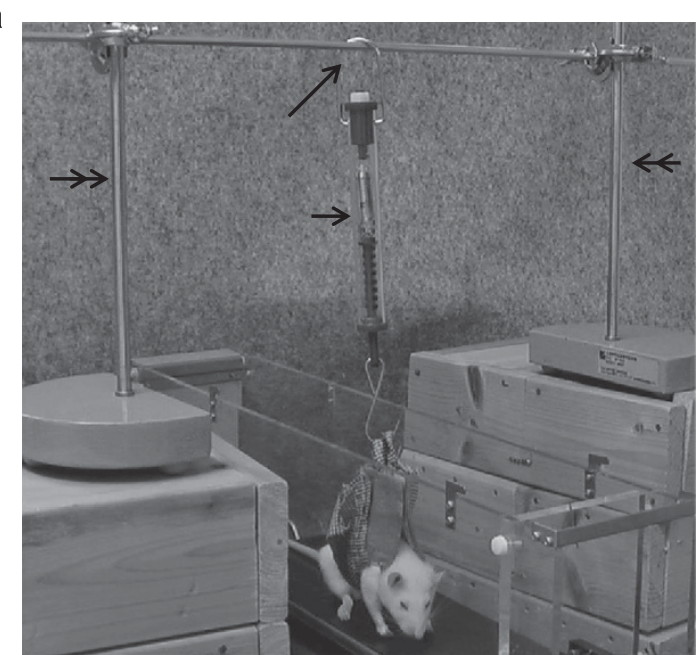

b

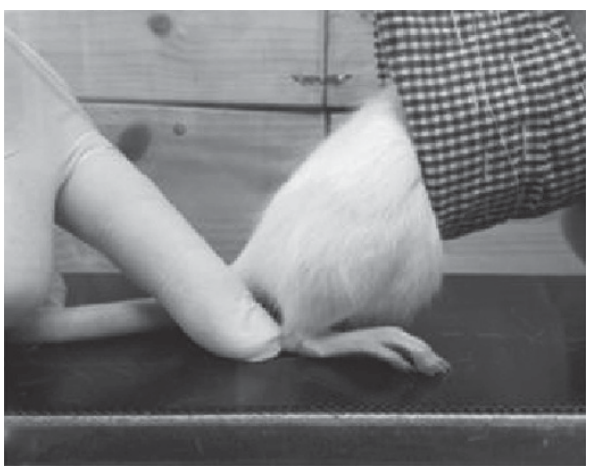

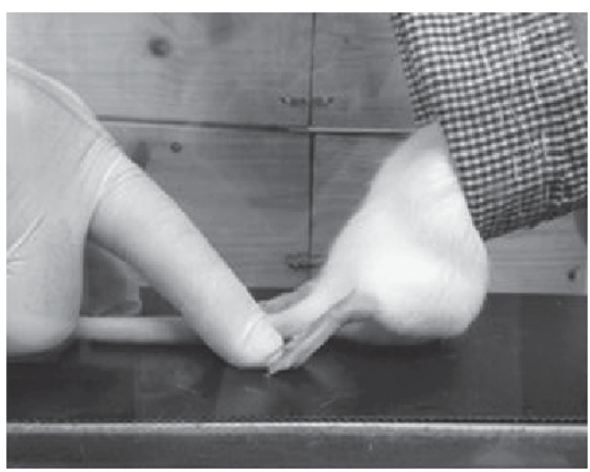

Figure 1 Treadmill training of rats. (a) Overview of treadmill training of rats. Rats were supported by a harness, the top of which was connected to a lever (spring) scale (short arrow), which was fixed to a steel bar (long arrow) bridging between two poles (double arrows) set on the left and right sides of the treadmill. (b) Position of hind paws in mid-stance during forced training by PP. (c) Position of hind paws in mid-stance during forced training by DP. 
a

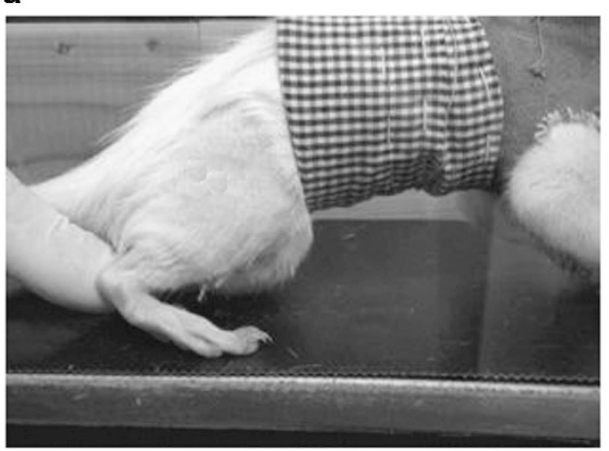

c

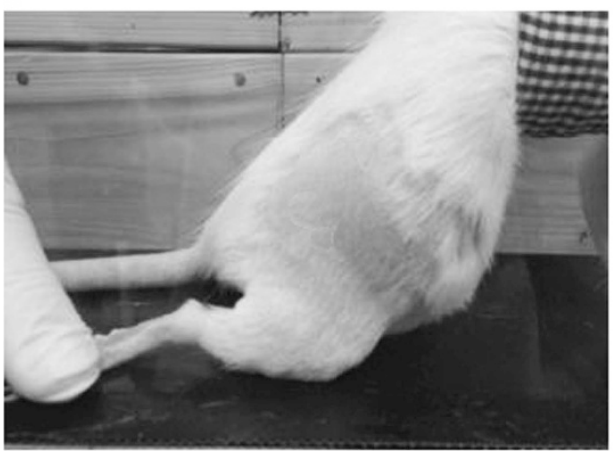

b

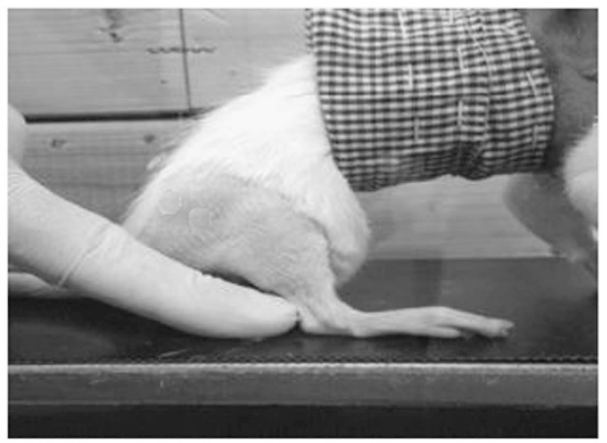

d

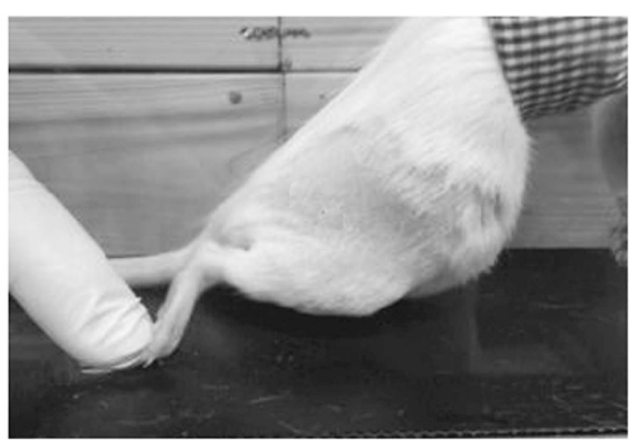

Figure 2 Forced step training by PP and DP. (a) The toe-off position of a hind paw in forced training by PP. The examiner puts his finger behind the paw. (b) The heel-contact position of a hind paw in forced training by PP. The examiner pushes the paw forward with his finger. (c) The toe-off position of a hind paw in forced training by DP. The examiner puts his finger on the sole of the paw. (d) The toe-contact position of a hind paw in forced training by DP. The examiner pushes the paw forward with his finger.

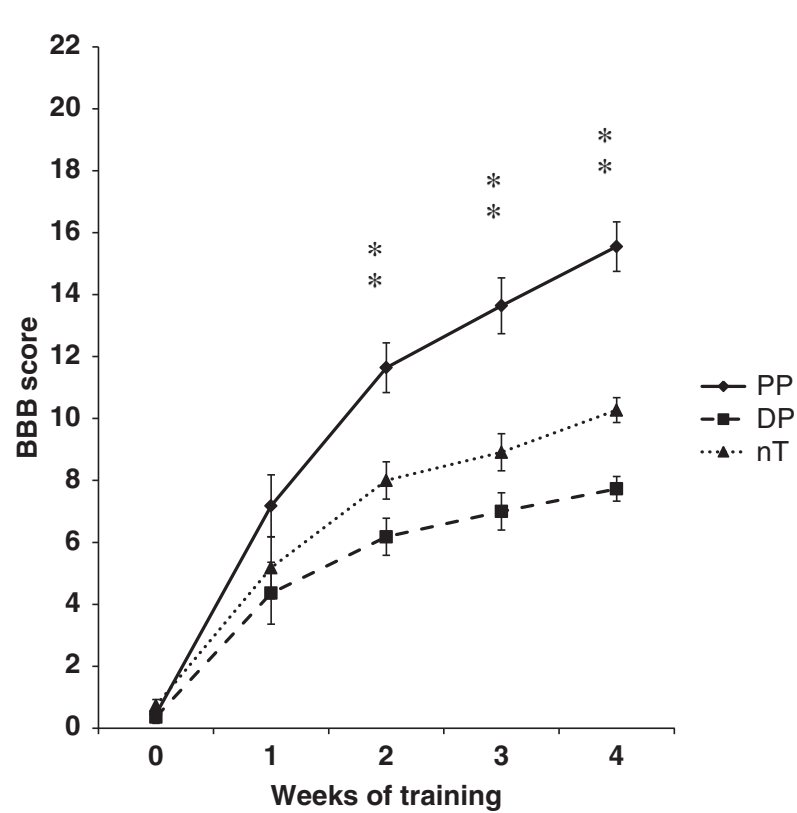

Figure 3 BBB scores. There were significant differences between the PP and $\mathrm{DP} / \mathrm{nT}$ groups at 2, 3 and 4 weeks of training $\left({ }^{*} P<0.05\right)$.

\section{Surgery}

Rats were anesthetized by the inhalation of isoflurane. The spinal cord with dura mater was exposed at the thoracic (Th) 8-9 level and crush-injured by dropping a 10-g metal weight from a height of $7.5 \mathrm{~cm}$ using an NYU impactor (New Brunswick, NJ, USA). As mentioned in the previous study, ${ }^{1}$ dropping a weight from a height of $5 \mathrm{~cm}$ tended to yield little difference between the experimental and control groups 4 weeks after injury. Therefore, the method of dropping a weight from a height of $7.5 \mathrm{~cm}$, the highest level of the NYU device, was employed in the present study.

After surgery, animals were given $3 \mathrm{ml}$ of saline subcutaneously to avoid dehydration. The rats were intramuscularly injected with antibiotics (gentacine, $3 \mathrm{mg} \mathrm{kg}^{-1}$ body weight, Schering Plough, Osaka, Japan) every day for 3 days.

\section{Training}

Rats were numbered randomly from No. 1 through 33. Rats Nos 1, 2 and 3 were assigned to the PP, DP and nT groups, respectively, and the remainder were assigned consecutively with every three rats to each group in the same manner. Treadmill training began 1 week after SCI and was conducted on 5 consecutive days per week for 4 weeks. Throughout the experiment, every effort was made to reduce the stress of the rats. The training was performed for 20 min per day by partial body weight support with a cloth harness (Figures 1 and 2). Three types of harnesses were prepared to adjust for the growth of rats over the 4 weeks of the experiment. The harness covered the thorax and upper half of the abdomen. The top of the harness was connected to a lever (spring) scale, which was fixed to a steel bar bridging between poles that were set on the left and right sides of the treadmill (Muromachi MK-685, Tokyo, Japan; Figure 1a). The scale of the lever connected with the harness was adjusted to $40 \%$ of the body weight of the rat. Figures $1 \mathrm{~b}$ and $\mathrm{c}$ show the mid-stance of hind paws in the PP and DP groups, respectively (see below for the forced training by PP and DP). The level of weight support (40\%) was not changed throughout the experiment.

The treadmill speed was $6.3 \mathrm{~m} \mathrm{~min}^{-1}$ in the first and second weeks and $8.6 \mathrm{~m} \mathrm{~min}^{-1}$ in the third and fourth weeks of training. An examiner assisted rats to take a step forward based on the frequency of a metronome. The metronome was set at 82 and 84 for treadmill speeds of 6.3 and $8.6 \mathrm{~m} \mathrm{~min}^{-1}$, respectively.

In the PP group, the rat was forced during every single step to walk quadripedally with the soles of the hind paws placed on the belt of the 
treadmill: an examiner used his finger to touch the heel in the toe-off position and then pushed it forward to the heel-contact position (Figures 2a and b). In the DP group, the rat was forced during every single step to walk quadripedally with the back of the hind paws and toes placed on the belt of the treadmill: an examiner used his finger to touch the sole of the paw in the toe-off position and pushed it forward to the toe-contact position (Figures $2 \mathrm{c}$ and $\mathrm{d}$ ). Rats were not forced to step on the treadmill in the nT group.

\section{Evaluation of locomotor behaviors}

Locomotor behaviors were evaluated based on BBB scores every week during the training period. Locomotor behaviors of rats were observed as they walked freely on a $1-\mathrm{m}^{2}$ mat for $5 \mathrm{~min}$ by two researchers not familiar with the experiment and, at the same time, recorded with a video camera (Panasonic HDC-HS9, Osaka, Japan). Locomotor behaviors were also assessed using images recorded with a video camera by the same researchers. Thus, the BBB scores were estimated both at the site and on a screen by examiners who were not informed of the experiments. The estimations were presented in a graph (Figure 3).

\section{Dorsiflexion angle of the hind paws}

This parameter was taken into account to evaluate the locomotor activity of the hind limbs. The measurement was performed at the end of the 4-week training. The hair on the lateral side of the right hind limb was shaved off, and black marks were placed on the patella, lateral malleolus and fifth metatarsal joint
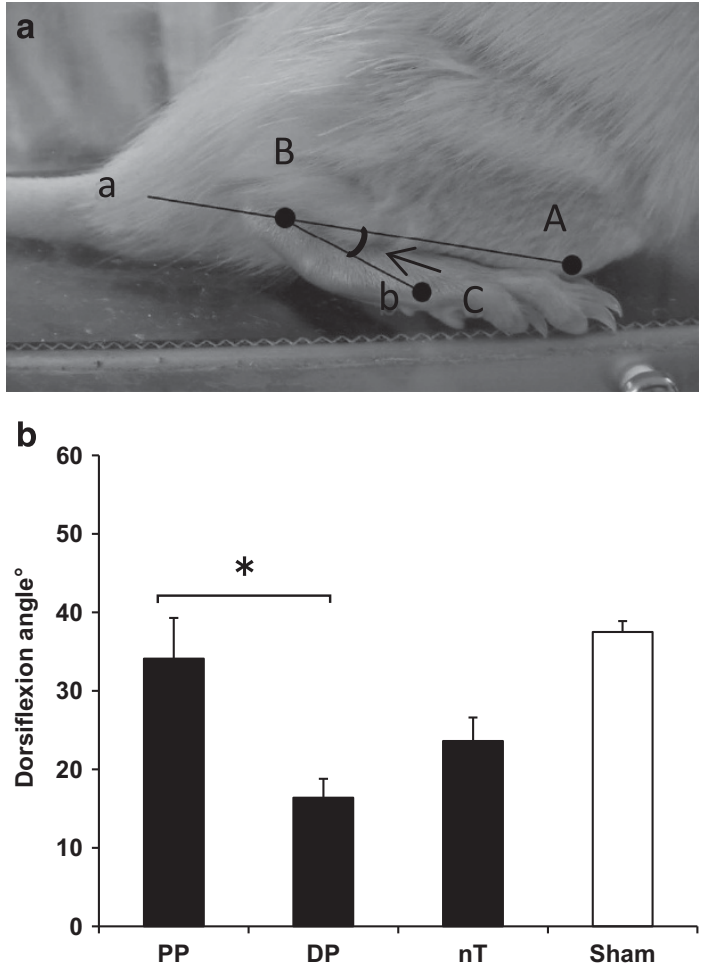

Figure 4 Dorsiflexion angle of the hind paw. (a) The photograph shows the dorsiflexion angle of the hind paw. The knee joint (A), lateral malleolus (B) and tip of the fifth metatarsalis (C) are marked with a circle. The line (a) drawn from the knee joint to the lateral malleolus served as the baseline. The dorsiflexion angle (arrows) was defined as the angle between this line and the line (b) drawn from the lateral malleolus to the tip of the fifth metatarsal bone. (b) The graph shows the dorsiflexion angles of hind paws. The maximum angle of hind paw dorsiflexion in the stance phase was regarded as the dorsiflexion angle. The dorsiflexion angle was $34.1 \pm 5.2$ degrees in the PP group, $16.4 \pm 2.4$ degrees in the DP group and $23.6 \pm 3.0$ degrees in the $\mathrm{nT}$ group at 4 weeks of training. The sham-operated group showed $37.5 \pm 1.4$ degrees. There was a significant difference between PP and DP groups ( $\left.{ }^{*} P<0.05\right)$. with a standard color marker. A rat walking on a stationary treadmill was recorded in the stance phase from the right side with a video camera, and the recorded images were analyzed on a computer. A line drawn from the knee joint to the lateral malleolus served as the baseline. The dorsiflexion angle was defined as the angle between this baseline and a line drawn from the lateral malleolus to the fifth metatarsal joint (Figure 4a). The maximum angle of dorsiflexion of the hind paw measured in the stance phase of stepping was used as the dorsiflexion angle.

\section{Footprints}

This parameter was measured to evaluate the locomotor activity of the hind limbs. The measurement was performed at the end of the 4-week training. India ink was applied to the soles of the hind paws, and rats were made to walk $2 \mathrm{~m}$ in total on white paper attached to the belt of the stationary treadmill (Figure 5a). The step length and step width over more than 20 steps were measured with a tape measure. In addition, to assess the stability of stepping, the angle between the line connecting the successive hind paw marks and the line showing the walking direction of rats was evaluated and defined as the stride angle (Figure $5 \mathrm{~b}$ ). The angle was measured with a goniometer.

\section{Histology and immunohistochemistry}

For histological and immunohistochemical examinations, rats were anesthetized at the end of the experiment (4 weeks of training) by the inhalation of isoflurane and were perfused intracardially with $50 \mathrm{ml}$ of phosphate-buffered saline, followed by $200 \mathrm{ml}$ of a fixative containing $2 \%$ paraformaldehyde in $0.1 \mathrm{~m}$ phosphate buffer ( $\mathrm{pH}$ 7.4). Specimens were immersed in 10, 20 and $30 \%$ sucrose solution ( 0.1 м phosphate buffer) for 1 day in each solution, embedded in OCT (Optimal Cutting Temperature; Funakoshi, Japan) compound and cut coronally or horizontally into 10 - $\mu \mathrm{m}$-thick frozen sections with a cryostat.

For histology, horizontal sections were stained with hematoxylin and eosin. Tissue recovery and cavity formation at the lesion site were examined. The cavity volume was measured in hematoxylin and eosin sections of three rats from each group as follows: the areas of cavities were measured on sections using software (Release $4.5 \mathrm{SP} 1$ ) in micrographs (Axio Vision, Carl Zeiss, Oberkochen, Germany.) and multiplied by the thickness of the section $(10 \mu \mathrm{m}$ for each section). When the sections were used for immunohistochemistry, the values of corresponding sections were deduced from adjacent sections and were added to the total value of the cavity volume. All values were totaled to obtain the cavity volume. The volume of the spinal cord at the lesion site was measured as follows: the average diameter of the spinal cord at the lesion site was obtained from three levels: at the epicenter of the lesion, at the level $1.5 \mathrm{~cm}$ rostral to and at the level $1.5 \mathrm{~cm}$ caudal to the epicenter. The area of the crosssection of the spinal cord at the lesion site was calculated using the average diameter. The volume was calculated by multiplying this cross-section by $3 \mathrm{~cm}$ (the length of the spinal cord). The relative cavity volume (\%) was calculated by dividing the cavity volume by the spinal cord volume at the lesion site.

For immunohistochemistry, after washing with phosphate-buffered saline and blocking with a blocking agent, sections were incubated overnight with a solution containing the following primary antibodies: anti-glial fibrillary acidic protein monoclonal antibody (1:300; Sigma-Aldrich, St Louis, MO, USA) for astrocytes or antineurofilament $200-\mathrm{kD}$ rabbit polyclonal antibody (1:100; Chemicon, Temecula, CA, USA) for axons. After washing, the sections were incubated overnight with the following secondary antibodies: Cy-3-labeled antimouse IgG antibody (1:1000; Amersham Bioscience, Amersham, UK) for astrocytes or fluorescein isothiocyanate-labeled anti-rabbit IgG antibody (1:1000; Amersham Biosciences) for axons. Sections were mounted with a cover glass and examined with a fluorescence microscope (Axio Imager MI; Carl Zeiss).

The density of regenerating axons at the lesion was evaluated. Clearly immunostained dots or strands were, regardless of their sizes, identified as axons, as shown in Figure 8e. A number of axon profiles within the astrocytedevoid areas were measured within the regions both $500 \mu \mathrm{m}$ rostral and caudal to the epicenter of the lesion in micrographs of longitudinal sections. The areas of astrocyte-devoid regions including cavities at the same levels were measured using the software ImageJ (NIH, Bethesda, MD, USA). The density of axons was calculated by dividing the number of axon profiles by the areas of 

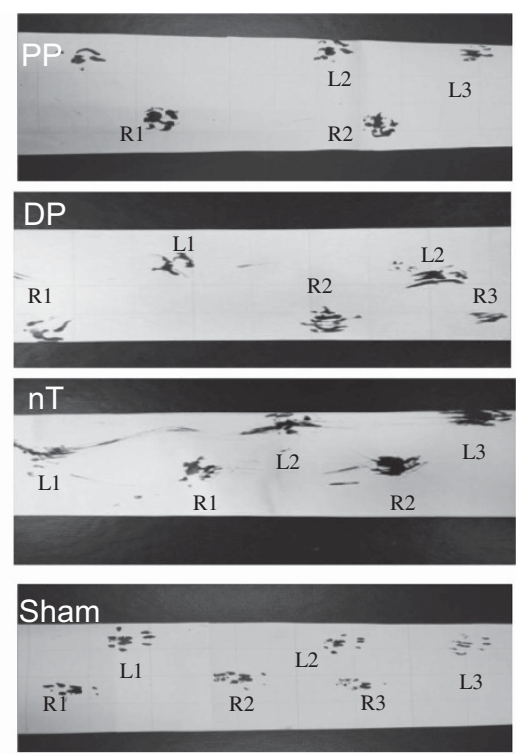

walking direction

b

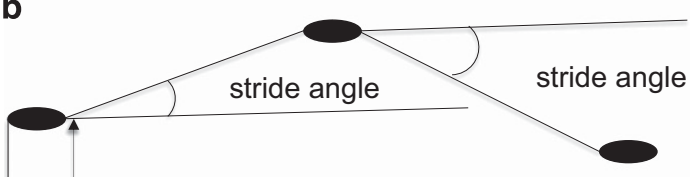

step width

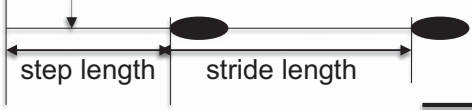

walking direction

Figure 5 Footprints. (a) These photographs show footprints of PP, DP, nT and sham-operated groups. The letters ' $L$ ' and ' $R$ ' mean footprints of left and right hind paws, respectively. (b) This drawing shows the step length, step width and stride angle. The angle between the line connecting successive hind paw marks and the line showing the walking direction of rats was defined as the stride angle.

astrocyte-devoid regions. This measurement was taken at three different levels of the spinal cord in three rats.

The areas of astrocytes were evaluated as follows: immunostained astrocytes were identified as shown in Figure 8f, and astrocyte areas were measured within the regions both $500 \mu \mathrm{m}$ rostral and caudal to the epicenter of the lesion in micrographs of longitudinal sections. The relative astrocyte areas were calculated by dividing the values of astrocyte-immunostained areas by the total spinal cord area at the same site. This measurement was taken at three different levels of the spinal cord of three rats.

\section{Statistics}

All data except axon density and astrocyte areas were analyzed by one-way analysis of variance followed by post hoc analysis using Tukey's test. Axon density and astrocyte areas were analyzed by Student's $t$-test. Significance was accepted at $P<0.05$.

\section{RESULTS}

Locomotor assessment of the BBB score

The BBB scores before training were in the range of $0.4 \sim 0.7$ points. Rats in the PP group showed an elevation of the average BBB scores from $0.5 \pm 0.2$ (mean \pm s.e.m.) points before training to $7.2 \pm 1.0$,
$11.6 \pm 0.8,13.6 \pm 0.9$ and $15.6 \pm 0.8$ points after $1,2,3$ and 4 weeks of training, respectively. Rats in the DP group showed an elevation from $0.4 \pm 0.2$ points before training to $4.4 \pm 1.1,6.2 \pm 1.3,7.0 \pm 1.3$ and $7.7 \pm 1.3$ points after $1,2,3$ and 4 weeks of training, respectively. There were significant differences between PP and DP after 2, 3 and 4 weeks of training (Figure 3). Rats in the $\mathrm{nT}$ group showed $0.7 \pm 0.2$, $5.2 \pm 1.0,8.0 \pm 0.6,8.9 \pm 0.6$ and $10.3 \pm 0.4$ points after $1,2,3$ and 4 weeks of training, respectively. There were significant differences between PP and nT groups at these time points.

\section{Dorsiflexion angle of hind paws}

All 11 rats in the $\mathrm{PP}$ and $\mathrm{nT}$ groups, respectively, and 7 rats in the DP group underwent measurement of the dorsiflexion angle of the hind paws (Figure 4a). Four out of the total of eleven in the DP group did not recover sufficiently to be able to walk with the paw sole placed on the treadmill belt. The dorsiflexion angle was $34.1 \pm 5.2$ (mean \pm s.e.m.) degrees in the PP group, $16.4 \pm 2.4$ degrees in the DP group and $23.6 \pm 3.0$ degrees in the $\mathrm{nT}$ group at 4 weeks of training (Figure $4 \mathrm{~b}$ ). The sham-operated group showed $37.5 \pm 1.4$ degrees. The PP group showed the same level of flexion as the sham-operated group. There was a significant difference between PP and DP groups. DP and nT groups exhibited smaller degrees of flexion than the PP or sham-operated group.

\section{Footprints}

All 11 rats in the PP and nT groups, respectively, and 7 rats in the DP group underwent the assessment of footprints (Figure 5). Four out of the total of eleven could not walk with the paw sole placed on the treadmill belt in the DP group. Footprints were examined at 4 weeks after training. The step length was $4.8 \pm 0.2$ (mean \pm s.e.m.), $4.1 \pm 0.3$, $3.8 \pm 0.3$ and $4.8 \pm 0.4 \mathrm{~cm}$ in the PP, DP, nT and sham-operated groups, respectively (Figure $6 \mathrm{a}$ ). The $\mathrm{PP}$ group showed a value similar to that of the sham-operated group. The step length of DP and nT groups tended to be shorter than that of PP and sham-operated groups. There was no significant difference in the step length among these groups. There was a tendency whereby the step length values of the PP group were similar to those of the sham-operated group.

The step width was $5.1 \pm 0.5,5.4 \pm 0.6,3.7 \pm 0.4$ and $4.1 \pm 0.1 \mathrm{~cm}$ in the PP, DP, nT and sham-operated groups, respectively (Figure 6b). There was no significant difference in the step width between PP and DP groups.

The stride angles were $5.1 \pm 1.4$ (R, right paw) and $5.1 \pm 0.9$ (L, left paw), $8.9 \pm 2.8(\mathrm{R})$ and $13.7 \pm 4.9(\mathrm{~L})$ and $16.9 \pm 3.5(\mathrm{R})$ and $17.8 \pm 4.0$ (L) in the PP, DP and nT groups, respectively (Figure 6c). The shamoperated group showed $4.0 \pm 0.5$ for both the left and right paws. The PP group showed values similar to those of the sham-operated group. DP and nT groups showed a large stride angle, indicating that their gait was unstable, staggering in appearance. There was a significant difference between PP and nT groups on both the left and right sides and between PP and DP groups on the left side.

\section{Cavity formation}

The cavity volume at the lesion site was measured in hematoxylin and eosin sections (Figure 7a). The representative images of each group are presented in Figure 7a. The cavity volume as a percentage of the spinal cord volume at the lesion site was calculated for each group. The values were $10.3 \pm 2.1 \%$ (mean \pm s.e.m.), $31.0 \pm 2.0 \%$ and $28.2 \pm 4.9 \%$ for $\mathrm{PP}, \mathrm{DP}$ and $\mathrm{nT}$ groups, respectively (Figure $7 \mathrm{~b}$ ). There was a significant difference between PP and DP groups, and between PP and nT groups, indicating that spinal cord tissue recovery was more enhanced in the PP than in the DP group. 

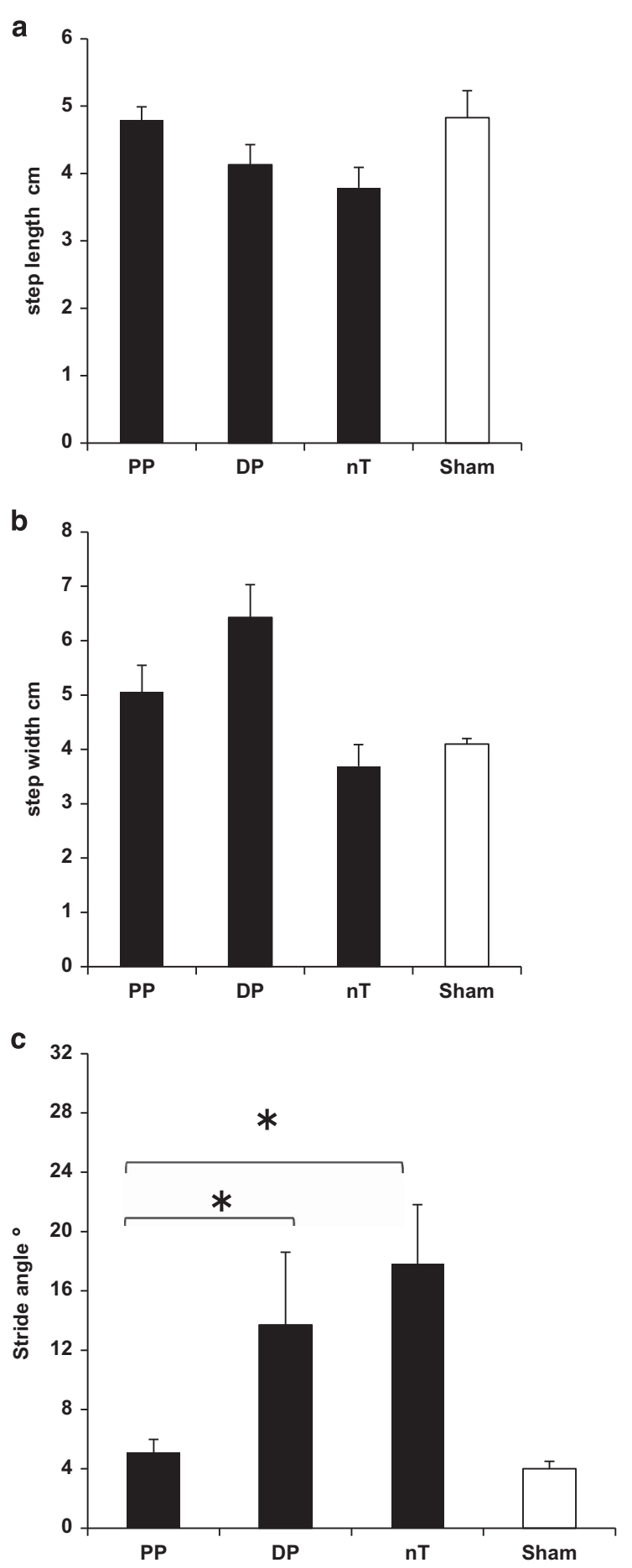

Figure 6 Graphs of step length, step width and stride angle. (a) Step length. There was no significant difference between PP and DP groups. The values for the PP group are close to those of the sham-operated group. (b) Step width. There was no significant difference between PP and DP groups. (c) Stride angle for the left paws. There was a significant difference between $\mathrm{PP}$ and DP groups and between PP and $\mathrm{nT}$ groups $\left({ }^{*} P<0.05\right)$. The values of the PP group are close to those of the sham-operated group.

\section{Astrocytes and axonal extension at the lesion}

Immunohistochemistry showed that astrocytes bordering the lesion did not migrate or extend their processes into the lesion, leaving astrocyte-devoid areas at the lesion site (Figure 8a). Some loose tissues were located within the astrocyte-devoid areas of the PP and DP groups. Cavities were a part of such astrocyte-devoid areas, in which no tissues were present as shown in the DP group.

Many axons were found extending through such loose tissues in the PP group, whereas axons are found only scarcely in the DP group (Figure $8 \mathrm{~b}$ ). The axon density was $1260 \pm 266 \mathrm{~mm}^{-2}$ in the PP group and $293 \pm 129 \mathrm{~mm}^{-2}$ in the DP group (Figure 8c). As cavities occupied a larger space in the DP group than in the PP group, the amount of some loose tissues in the astrocyte-devoid areas was limited in the DP group, resulting in a small number of axons extending in the astrocyte-devoid areas in the DP group. The findings of the nT group were similar to those of the DP group. The astrocyte areas in the spinal cord lesion were $8.1 \pm 0.6 \%$ in the PP group and $4.6 \pm 1.2 \%$ in the DP group (Figure 8d).

\section{DISCUSSION}

The present study demonstrated that the PP group showed more marked locomotor improvement and tissue recovery, including axonal extension, at the spinal cord lesion than the DP or the $\mathrm{nT}$ group. Rats had $40 \%$ of their body weight supported during forced treadmill training. An accurate estimate of body weight loading on the hind paws was not possible in the present study.

\section{Locomotor improvement}

Stepping patterns and dorsiflexion angles of hind paws were closer between the PP group and the sham-operated group than the DP group. Although the SCI employed in the present study was severe, the $\mathrm{BBB}$ scores increased to as high as $15-16$ points. Rats with such high BBB scores showed almost normal stepping patterns, suggesting the reason why there was little difference in stepping parameters examined in the present study between the PP and sham groups.

It is suggested that PP is critical for rats to recover from SCI. The locomotor recovery/score improvement correlates with the ability to perform PP with a higher consistency. Sensory information from the soles of the hind paws, movements of the hind paws and hind limb joints and body weight loading during forced training may contribute to activate locomotor functions in the PP group. DP of the hind paws and toes did not contribute to locomotor improvement, indicating that (a) information from the dorsum of the paws and toes and (b) the limited activities of the hind paws did not elicit any effective locomotor functions in the spinal cord.

Battistuzzo et al. ${ }^{6}$ stated in their review that BWST training produced positive locomotor outcomes in $75 \%$ of studies in rats. In contrast, robotic-assisted training was only successful in $25 \%$ of rat studies. Locomotor recovery was greater in the assist-as-needed group than in the fully assisted group of robotic training, indicating that manual assistance in stepping is beneficial for the improvement of locomotor movements. The technique of forced PP stepping in the present study would correspond to manual BWST training in humans, suggesting that manual BWST training may be beneficial, as opposed to fully assisted robotic BWST training.

\section{BBB scores}

The effectiveness of treadmill training was reported in other studies. ${ }^{5,12}$ Multon et al. ${ }^{13}$ performed an experiment in which spinal cord-injured rats were made to walk quadrapedally on a treadmill while the body was manually supported. The rats showed an elevation of $\mathrm{BBB}$ scores from 2 points before training to 10 points after 5 weeks of training. These values are comparable to those of the PP group in this study. It was reported that BBB scores were 15 points at 3-4 weeks after injury in mild-moderately injured rats without training. ${ }^{14}$ In the 
a
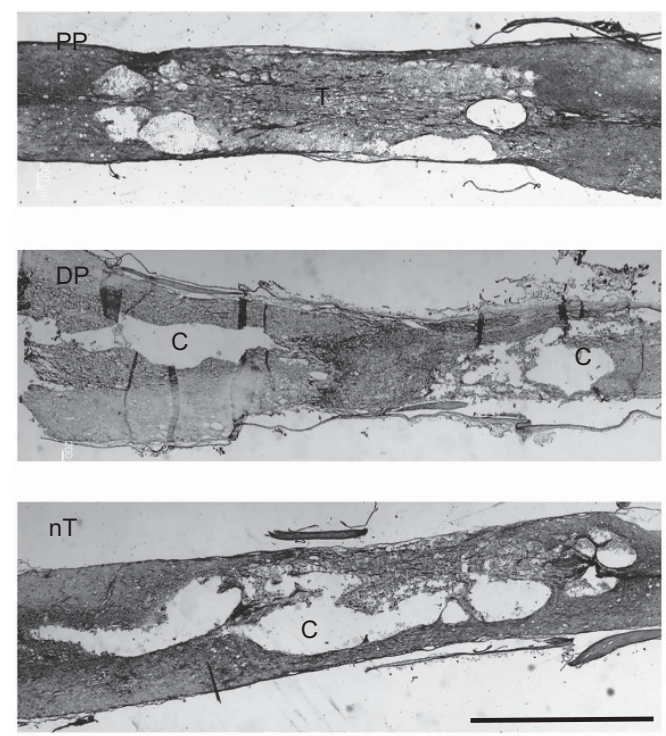

b

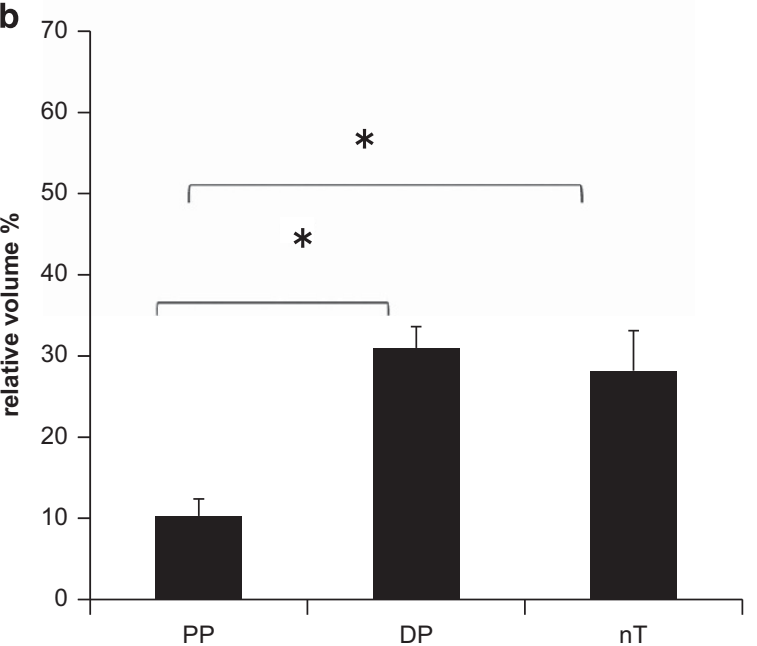

Figure 7 Cavity formation observed in horizontal sections of the spinal cord lesion. HE staining. (a) Representative micrographs of each group. Tissue (T) has recovered within the spinal cord lesion in the PP group, whereas cavities (c) of various sizes are prominent in the spinal cord lesions of the DP and nT groups. Scale bar: $500 \mu \mathrm{m}$. (b) This graph shows relative cavity sizes at spinal cord lesions in each group. The relative cavity sizes were calculated by dividing the cavity volume by the spinal cord volume at the lesion site. There were significant differences between PP and DP/nT groups (*).

present study, BBB scores were $8.9-10.3$ points at 3-4 weeks post injury in the $\mathrm{nT}$ group of severely injured rats. On the other hand, there is a report ${ }^{12}$ that BBB scores were $14,11-12$ and 9.3-9.6 points for mildly, moderately and severely injured rats, respectively, after 4-week training. Multon et al. ${ }^{13}$ showed that BBB scores were 9.5 points for trained and 6 points for untrained rats at 4 weeks post injury in severely injured rats. BBB scores of 15.6 points for trained and 10.3 points for untrained rats at 5 weeks post injury in the present study indicate that the injury adopted in the present study might be, as a result, less severe than that in these studies.

Although a precise estimate of body weight loading is not possible, inputs from paw soles loaded with body weight are considered to make a major contribution to locomotor improvement. The limited movements of hind paws and hind limb joints had little or no effects on locomotor recovery in the DP group. The BBB values of the $\mathrm{nT}$ group are compatible with those of the DP group. From the standpoint of BBB scores, the DP group was under the same condition as the nT group.

\section{Stepping patterns and dorsiflexion of hind paws}

The fact that the PP group was closer to the sham-operated group than the DP group suggests that stepping patterns were in the process of improvement in the PP group.

There were significant differences in the stride angle between PP and DP and between PP and nT groups. These indicate more prominent staggering gaits in the DP and $\mathrm{nT}$ than in the PP group. PP of the hind paws may contribute to coordinated and straightforward stepping of the hind limbs.

The values of dorsiflexion in the stance phase in the PP group were similar to those in the sham-operated group and larger than those in $\mathrm{DP}$ and $\mathrm{nT}$ groups. The importance of flexion and extension of the ankle joint was referred to in previous studies. ${ }^{14}$ It is suggested that PP enhanced the supporting function of the ankle joint in SCI.

\section{Central pattern generator}

Treadmill training aims, in part, to promote neural circuit connectivity by activating the central pattern generator (CPG). CPG, located inherently in the spinal cord, is not restricted to a limited region of the spinal cord but is distributed throughout it. Grasso et al. ${ }^{8}$ reported that locomotor responses elicited by treadmill training in SCI patients may depend on a plastic redistribution of activity across most of the rostrocaudal extent of the spinal cord. Rossignol et al. ${ }^{15}$ reviewed the role of CPG in locomotor recovery after incomplete SCI. It has been recognized that partial body weight support with the loading of weight on the hind feet is important for locomotor training. ${ }^{8,16}$ Sensory inputs were reported to be important in the body weight-supported treadmill training of patients. ${ }^{17}$ Dietz et al. ${ }^{7}$ reported that afferent inputs from hip joints and inputs from load receptors had a crucial role in the generation of locomotor activity in the presence of complete para/tetraplegia of the human spinal cord. It is possible that movements of hind limb joints, especially those of hip joints, might be greater or more dynamic in the PP than in the DP group. In addition, the center of gravity of the body might function more effectively in the PP than in the DP group. These factors may also have contributed to the improvement of locomotor movement.

BWST training by PP of the hind paws may elicit sensory inputs from the paw sole, partly contributing to the development of the CPG function. On the contrary, inputs from the back of the paw and toes might not contribute to improvement of the locomotor function. It is considered that the paw sole, but not the back of the paw and toes, serves as a load receptor in gait movement.

\section{Tissue recovery and axonal extension}

The present study indicates that forced training by PP with body weight bearing is effective for tissue recovery after SCI. It is suggested that astrocytes could not migrate or extend their processes into the lesion, leaving an astrocyte-devoid area at the lesion site. This suggests that the loose tissues occupying the astrocyte-devoid areas at the lesion might not belong to CNS tissues. It is conceivable that the tissue within the astrocyte-devoid area is not an original glial tissue but is a non-glial one that had been formed after injury. Axons found in the astrocyte-devoid tissue might be those that had grown following the injury. ${ }^{18}$ It has been reported that exercise promotes tissue formation and axonal extension in lesions of SCI. ${ }^{5,19}$ The severity of SCI is considered to be similar between the PP and DP groups, judging from 

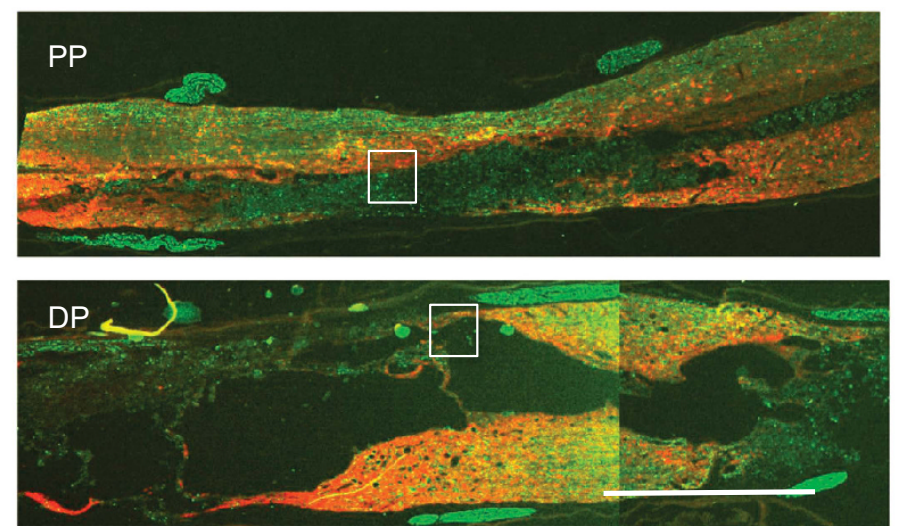

b
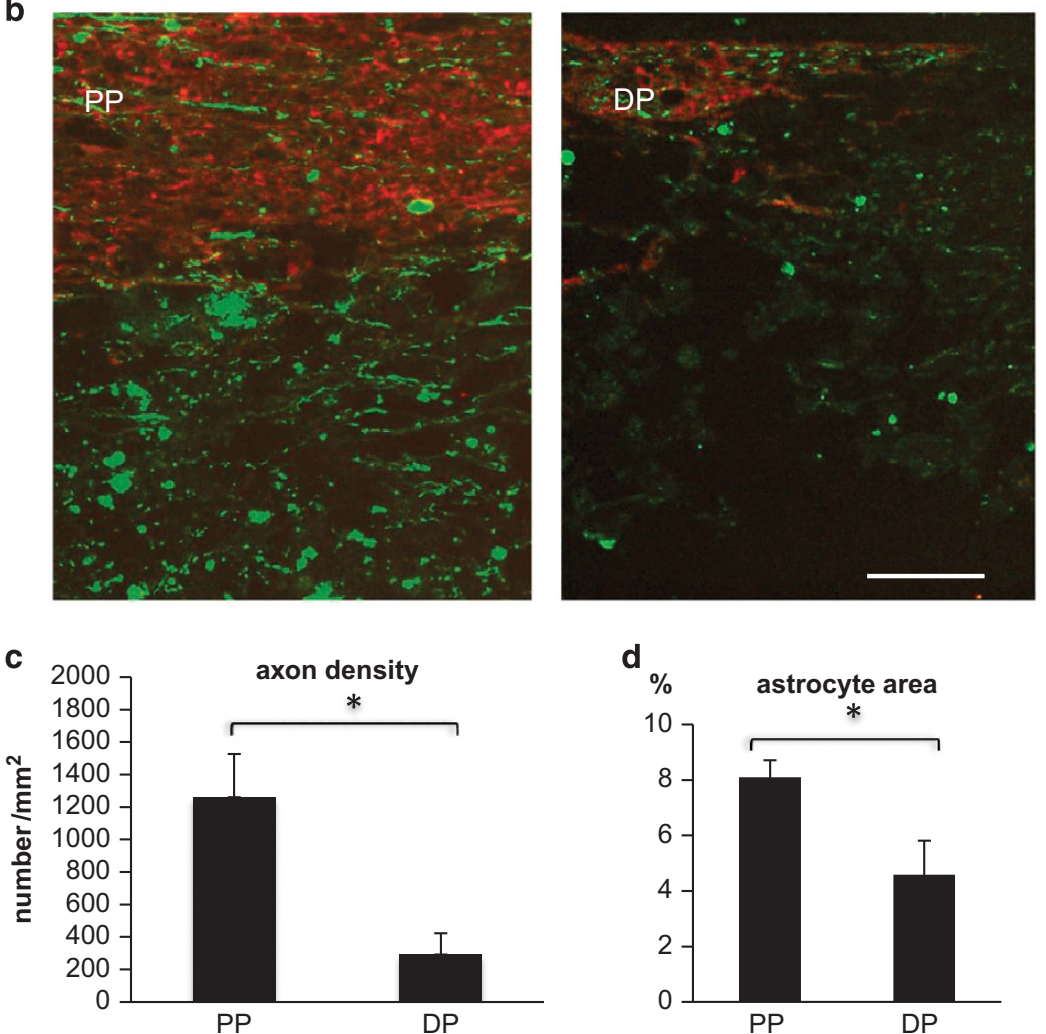

e

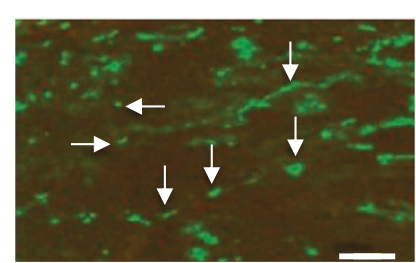

f

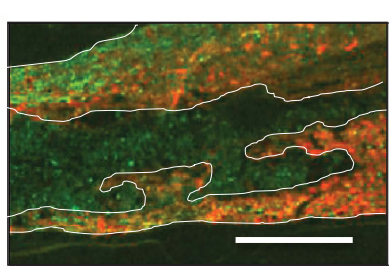

Figure 8 Immunohistochemistry showing axonal extension in the spinal cord lesions. Representative micrographs from the PP and the DP groups are shown. (a) These low-powered immunohistochemical micrographs show the location of astrocytes and axons in the spinal cord lesion. Astrocytes are stained red, and axons are green. There are areas devoid of astrocytes in the lesions, where green-stained tissue is present. The vacant spaces in the astrocyte-devoid areas are cavities. There are large cavities in the DP group. The green-stained tissues in the astrocyte-devoid areas are considered to partly correspond to the loose tissue occupying the lesion in the hematoxylin and eosin (HE) sections (Figure 7a). Scale bar: $500 \mu$ m. (b) High-powered micrograph of immunohistochemistry of the spinal cord lesions. These enlarged micrographs were taken of the sections adjacent to those shown in a. The area in the adjacent section corresponding to the site marked by the rectangle was enlarged in each group. There are numerous axons extending longitudinally (brightgreen fibers) in the astrocyte-devoid area of the PP group. In contrast, axons were found only scarcely in the astrocyte-devoid area of the DP group. The host astrocyte region (red) at the periphery of the lesions contains some axons (green) in both the PP and the DP groups. Scale bar: $25 \mu \mathrm{m}$. (c) Axon density. There was a significant difference between PP and DP groups $(*)$. (d) Astrocyte area. There was a significant difference between PP and DP groups (*). (e) Clearly immunostained dots or strands were, regardless of their sizes, identified as axons. Arrows point to some axons. Scale bar: $25 \mu \mathrm{m}$. (f) Astrocyte areas were measured as shown in this micrograph. Scale bar: $500 \mu \mathrm{m}$. 
the BBB scores of $0.5 \pm 0.3$ and $0.4 \pm 0.2$ for PP and DP groups, respectively. During the course of training, cavity formation was reduced and axonal regeneration was promoted in the PP group as compared with the DP group. These tissue recoveries in the PP group may be due to the combinatorial effects of PP stepping on the reduction or the retardation of tissue degeneration and/or the enhancement of axonal extension in injured spinal cord. Although the mechanisms for these tissue recoveries in spinal cord lesions are not fully understood, this tissue organization and axonal outgrowth in the injured spinal cord is promising for rehabilitation therapy in humans.

\section{Limitations}

It was suggested that sensory inputs from the paw sole, but not the back of the paw, might contribute to the development of the CPG function and enhancement of tissue recovery including promoted axonal regeneration in the spinal cord. There was no information concerning the sensory inputs to the spinal cord in the PP and DP groups. Sensory inputs to the spinal cord during the forced locomotion by PP and DP should have been analyzed electrophysiologically.

The three-dimensional analysis and electromyography of hind limb movements would have enabled a more precise analysis of locomotion, leading to the identification of any differences in parameters of hind limb stepping between the PP and sham groups.

The promotion of nerve regeneration in the PP group is important. The mechanism for this phenomenon is not known. Synapse formation with regenerating axons should be studied for the establishment of neural connections in the injured spinal cord.

\section{DATA ARCHIVING}

There were no data to deposit.

\section{CONFLICT OF INTEREST}

The authors declare no conflict of interest.

\section{ACKNOWLEDGEMENTS}

This study was supported, in part, by Grants-in-Aid for Scientific Research from the Japanese Society for the Promotion of Science (No.20300122 and 23300125) to CI and Promotive Research Fund awarded from Aino University to $\mathrm{MH}$
1 Nakano N, Nakai Y, Seo TB, Homma T, Yamada Y, Ohno T et al. Effects of bone marrow stromal cell transplantation through CSF on the subacute and chronic spinal cord injury in rats. PLOS ONE 2013; 8: e73494.

2 Ohta M, Suzuki $\mathrm{Y}$, Noda $\mathrm{T}$, Ejiri $\mathrm{Y}$, Dezawa M, Katatoka $\mathrm{K}$ et al. Bone marrow stromal cells infused into the cerebrospinal fluid promote functional recovery of the injured rat spinal cord with reduced cavity formation. Exp Neurol 2004; 187: 266-278.

3 Suzuki Y, Ishikawa N, Omae K, Hirai T, Ohnishi K, Nakano N et al. Bone marrowderived mononuclear cell transplantation in spinal cord injury patients by lumbar puncture. Restor Neurol Neurosci 2014; 32: 473-482.

4 Dobkin BH, Harkema S, Requejo P, Edgarton VR. Modulation of locomotor-like EMG activity in subjects with complete and incomplete spinal cord injury. J Neurol Rehabil 1995; 9: 183-190.

5 Goldshmit Y, Lythgo N, Galea MP, Turnley AM. Treadmill training after spinal cord hemisection in mice promotes axonal sprouting and synapse formation and improves motor recovery. J Neurotrauma 2008; 25: 449-465.

6 Battistuzzo CR, Callister RJ, Callister R, Galea MP. A systematic review of exercise training to promote locomotor recovery in animal models of spinal cord injury. J Neurotrauma 2012; 29: 1600-1613.

7 Dietz V, Muller R, Colombo G. Locomotor activity in spinal man: significance of afferent input from joint and load receptors. Brain 2002; 125: 2626-2634.

8 Grasso R, Ivanenko YP, Zago M, Molinari M, Scivoletto G, Castellano V et al. Distributed plasticity of locomotor pattern generators in spinal cord injured patients. Brain 2004; 127: 1019-1034.

9 Varejao AS, Filipe VM. Contribution of cutaneous inputs from the hind paw to the control of locomotion in rats. Behav Brain Res 2007; 176: 193-201.

10 Zhang Y, Ji SR, Wu CY, Fan XH, Zhow HJ, Liu GL. Observation of locomotor functional recovery in adult complete spinal rats with BWSTT using semiquantitative and qualitative methods. Spinal Cord 2007; 45: 496-501.

11 Basso DM, Beattie MS, Bresnahan JC. A sensitive and reliable locomotor rating scale for open field testing in rats. J Neurotrauma 1995; 12: 1-21.

12 Nessler JA, De Leon RD, Sharp K, Kwak E, Minakata K, Reinkensmeyer DJ. Robotic gait analysis of bipedal treadmill stepping by spinal contused rats: characterization of intrinsic recovery and comparison with BBB. J Neurotrauma 2006; 23: 882-896.

13 Multon S, Franzen R, Poirrier AL, Scholtes F, Schoenen J. The effect of treadmill training on motor recovery after a partial spinal cord compression-injury in the adult rat. J Neurotrauma 2003; 20: 699-706.

14 Hillen BK, Yamaguchi GT, Abbas JJ, Jung R. Joint-specific changes in locomotor complexity in the absence of muscle atrophy following incomplete spinal cord injury. J Neuroeng Rehabil 2013; 10: 97.

15 Rossignol S, Barriere G, Alluin O, Frigon A. Re-expression of locomotor function after partial spinal cord injury. Physiology 2009; 24: 127-139.

16 Hesse S, Konrad M, Uhlenbrock D. Treadmill walking with partial body weight support versus floor walking in hemiparetic subjects. Arch Phys Med Rehabil 1999; 80: 421-427.

17 Beres-Jones JA, Harkema SJ. The human spinal cord interprets velocity-dependent afferent input during stepping. Brain 2004; 127: 2232-2246.

18 Ide C, Nakai Y, Nakano N, Seo TB, Homma Y, Ohta M et al. Bone marrow stromal cell transplantation for treatment of sub-acute spinal cord injury in the rat. Brain Res 2010; 1332: 32-47.

19 Houle JD, Cote MP. Axon regeneraion and exercise-dependent plasticity after spinal cord injury. Ann NY Acad Sci 2013; 1279: 154-163. 